\title{
Armazenamento e qualidade da noz macadâmia
}

\author{
Fernanda de Ávila ${ }^{1}$
}

\author{
Maria Cecília de Arruda Palharini
}

Marcos José Perdoná ${ }^{3}$

\begin{abstract}
RESUMO O cultivo e comercialização da macadâmia estão crescendo rapidamente no Brasil. As colheitas se concentram entre os meses de fevereiro a abril, porém, os melhores preços para a noz são alcançados no final do ano. $\mathrm{O}$ armazenamento nas propriedades pode ser uma estratégia para que os produtores consigam maiores preços de venda e tenham maior êxito nessa atividade, entretanto, existe o risco da perda da qualidade das nozes, o que acarretaria sérios prejuízos. Essa revisão objetivou agregar informações sobre o cultivo e armazenamento da noz macadâmia nas propriedades e sobre os métodos disponíveis para avaliar o sucesso do armazenamento nas condições brasileiras.
\end{abstract}

PALAVRAS-CHAVE:Macadamia integrifolia, pós-colheita, rancidez.

\section{Storage and quality of macadamia nuts}

\footnotetext{
${ }^{1}$ Graduanda de Farmácia, na Universidade Sagrado Coração (USC). feer.avila@gmail.com.

${ }^{2}$ Eng. Agrônoma, Doutora, Pesquisadora Científica da Agência Paulista de Tecnologia dos

Agronegócios (APTA), Bauru-SP. mcarruda@yahoo.com.br .

${ }^{3}$ Eng. Agrônomo, Doutor, Pesquisador Científico da Agência Paulista de Tecnologia dos

Agronegócios (APTA), Bauru-SP,marcosperdona@apta.sp.gov.br.
} 
ABSTRACT The cultivation and marketing of macadamia is rapidly growing in Brazil. The harvest are concentrated between the months from February to April, however, the best prices for the nut are achieved at the end of the year. Storage in the properties may be a strategy so that producers are able to higher sales prices and have greater success in this activity, however, there is a risk of loss of quality of the nuts, which would cause serious damage. This review aimed to add information on the cultivation and storage of macadamia nuts in the properties and the methods available to evaluate the success of storage in Brazilian conditions.

KEY-WORDS: Macadamia integrifolia, postharvest, rancidity.

\section{Almacenamiento y calidad de las nueces de macadamia}

RESUMENEI Cultivo y la comercialización de macadamia está creciendo rápidamente en Brasil. Los cosechas se concentran entre los meses de febrero a abril, pero, los mejores precios de nueces son alcanzados al final del año. El almacenamiento en las propiedades puede ser una estrategia para que los productores obtendrán mayores precios de venta y tiendrán un mayor éxito en esta actividad, sin embargo, existe el riesgo de pérdida de la calidad de los frutos secos, lo que causaría graves daños. Esta revisón apunta a agregar información sobre el cultivo y almacenamiento de nueces de macadamia en las propiedades y los métodos disponibles para evaluar el éxito de su almacenamiento en condiciones brasileñas.

PALABRAS CLAVE:Macadamia integrifolia, después de la cosecha, la rancidez

\section{INTRODUÇÃO}

As nogueiras-macadâmia (Macadamia ssp.) são árvores nativas das florestas tropicais da Austrália. A Macadamiaintegrifólia, espécie mais cultivada, teve seu desenvolvimento tecnológico no Hawaii (MCFADYEN et al., 2012). A utilização dessa noz é ampla. Ela pode ser consumida crua ou torrada e salgada, sendo muito utilizada em confeitaria, bolos, biscoitos, sorvetes e outros tipos de doces (MARTIN, 1992).

Considerada a mais saborosa noz do mundo (HUETT, 2004), essas amêndoas possuem um conjunto de componentes altamente nutritivos e são importantes para uma dieta saudável, promovendo boa saúde, longevidade e redução de doenças degenerativas.

No Brasil, esta nogueira chegou na década de 1940, mas ainda é pouco explorada (SCHNEIDER et al., 2012). No Estado de São Paulo, ocupa área de aproximadamente 2.200 ha (CATI, 2008) e,embora seu o cultivo tenha crescido, sua expansão encontra dificuldades por conta do elevado período de retorno do 
investimento (Perdoná et al., 2015). Já o consumo no mercado nacional cresce rapidamente (Perdoná \& Soratto, 2015b).

As colheitas estão concentradas entre os meses de fevereiro e abril (Sobierajski et al., 2007), entretanto, o maior consumo das nozes acontece no mês de dezembro, quando ocorrem os maiores preços. Assim, o armazenamento poderia gerar maior renda aos produtores, porém, esses temem a perda de qualidade do produto, pois há falta de informações confiáveis nesse segmento.

\section{OBJETIVO}

Considerando o potencial de expansão da cultura no país, e a possibilidade de ampliar a lucratividade dos produtores através da realização de vendas na entressafra,essa revisão objetivou agregar informações sobre o cultivo e armazenamento da noz macadâmia nas propriedades e sobre os métodos disponíveis para avaliar o sucesso do armazenamento nas condições brasileiras.

\section{A MACADÂMIA}

Os aborígines australianos sempre consumiram sua amêndoa, apesar disso, o inglês Walter Hill é considerado o descobridor da macadâmia. Dentre mais de 10 espécies do gênerodescritas, as espécies Macadamia integrifolia (Maiden e Betche) e Macadamia tetraphylla (L.)sãoas únicas exploradas economicamente (BRENES, 1983). A M. integrifolia teve seu desenvolvimento tecnológico no Hawaii Agricultural Experiment Station (HAES), onde foram selecionadas as principais cultivares plantadas hoje no mundo (PIMENTEL, 2007; MCFADYEN et al., 2012).

A utilização dessa noz é ampla. Ela pode ser consumida crua ou torrada e salgada, sendo muito utilizada em confeitaria, bolos, biscoitos, sorvetes e outros tipos de doces (MARTIN, 1992). Considerada a mais saborosa noz do mundo (SACRAMENTO, 1991; HUETT, 2004), a noz tem em sua composição aproximadamente $80 \%$ de óleo. Sua amêndoa é rica em óleos monoinsaturados e, graças às características nutricionais, é considerada excelente fonte energética. Os 
ácidos graxos mais encontrados em sua composição são: palmítico, palmitoléico (ômega-7), esteárico, oléico (ômega-9) e linoléico (ômega-6) (MARO et al., 2012), que são disputados pela indústria de cosméticos, para uso na composição de hidratantes, e por laboratórios farmacêuticos, como redutor dos níveis de colesterol (HUETT, 2004; SILVA et al., 2007). As amêndoas possuem um conjunto de componentes altamente nutritivos e são importantes para uma dieta saudável, promovendo boa saúde, longevidade e redução de doenças degenerativas. Em seus trabalhos, Sponchiato (2008) verificou que uma dieta que incluiu macadâmias reduziu em $6 \%$ os níveis de colesterol e colaborou na prevenção de doenças cardiovasculares.

\subsection{A MACADÂMIA NO BRASIL}

No Brasil, na década de 1940, o Instituto Agronômico de Campinas (IAC) iniciou os trabalhos de adaptação e melhoramento da espécie, tendo como base as cultivares HAES. Na década de 1970 o IAC lançou as primeiras cultivares adaptadas às condições climáticas brasileiras. Hoje, estes dois centros são responsáveis por produzir as cultivares mais utilizadas em nosso país, todos pertencentes à espécie M. integrifolia. São elas: HAES 344, HAES 660, HAES 741, HAES 816 e IAC 121, IAC 4-12B, IAC Campinas B, IAC 9-20 e IAC 4-20 (GARBELINI, 2009).

A macadâmia possui alto valor no mercado internacional e tem grande potencial no mercado interno, mas ainda é pouco explorada no Brasil (BOAS et al., 2012; MARO et al., 2012; PIO et al., 2012; SCHNEIDER et al., 2012). Levantamentos realizados pela CATI (2008) mostram que 199 produtores investiam no seu cultivo e a macadâmia era a principal nogueira cultivada no Estado de São Paulo, ocupando área de aproximadamente 2.200 ha. Embora o cultivo da nogueira-macadâmia tenha crescido nos últimos anos no Estado de São Paulo, sua expansão encontra dificuldades por conta do elevado período de retorno do investimento, condição que também poderá ser modificada pelo uso da irrigação e da consorciação com outras culturas (Perdoná et al., 2015). A irrigação e a consorciação com café arábica, por exemplo, reduziram o período de retorno do 
investimento da cultura da macadâmia em pelo menos três anos (Perdoná \& Soratto, 2015a).

O mercado nacional tem crescido rapidamente nos últimos anos, com isso, o parque industrial instalado opera com apenas metade da sua capacidade instalada pela falta da noz como matéria-prima (Perdoná \& Soratto, 2015b). Para Pimentel (2007), esse um imenso mercado interno inexplorado, pode ser fator de incremento no agronegócio nacional.

Para Pimentel et al. (2007), a fase de pós-colheita da macadâmia é de extrema importância, pois influencia diretamente na qualidade do produto. As colheitas estão concentradas entre os meses de fevereiro e abril (Sobierajski et al., 2007; Aparecido et al., 2014), entretanto, o maior consumo das nozes acontece no mês de dezembro, por ocasião das festas natalinas, quando também ocorrem os maiores preços para essas nozes. Apesar disso, o armazenamento dessa noz é pouco praticado pelos produtores, que temem a perda de qualidade do produto, pois há falta de informações confiáveis nesse segmento. As indústrias, por sua vez, armazenam suas nozes em silos, garantindo o abastecimento do mercado durante todo o ano. Ali, a umidade das amêndoas é baixada para próximo de $1 \%$, o que diminui a possibilidade da proliferação de fungos e preserva a qualidade das nozes.

\section{MÉTODOS DE ANÁLISE}

A qualidade pode ser definida como um conjunto de atributos que levam ou não à aceitação do produto pelo consumidor. Pode ser avaliada pelos seus principais atributos físicos, químicos, microbiológicos e sensoriais (Chitarra \& Chitarra, 2005).

\subsection{PRESENÇA DE FUNGOS}




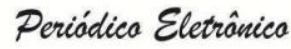 \\ Fórum Ambiental}

da Alta Paulista
Volume 11, Número 02, 2015

Campo, Agronegócio

e as Práticas Sustentáveis

Um estudo realizado por Schneider et al. (2012) mostra que, para se ter um desenvolvimento e uma produção adequados, a nogueira necessita de temperatura ótima entre 20 e $25^{\circ} \mathrm{C}$ e precipitação pluviométrica de $1000 \mathrm{~mm}$ anuais. Este tipo de ambiente é favorável para o desenvolvimento de fungos, que podem contaminar o fruto no campo, mas principalmente durante o seu armazenamento, e assim há consequente aparecimento de micotoxinas.

Walton e Walace (2010) consideram que o tempo de armazenamento da macadâmia na propriedade pode afetar sua qualidade. Estes autores recomendam que as nozes tenham umidade menor que $10 \%$ ao saírem das propriedades, para que o produto seja transportado para o processamento sem comprometimentos por fungos.

Nos países em desenvolvimento, a incidência de alimentos contaminados por micotoxinas ainda é grande. Micotoxinas são metabólitos secundários de fungos e podem ser produzidas no campo ou durante 0 armazenamento de alimentos. A ingestão pode causar micotoxicoses em animais e humanos, que podem descrever episódios agudos ou crônicos da doença. Cerca de $25 \%$ do que é colhido no mundo pode ter crescimento de fungos e consequentemente produção de micotoxinas, e a sua redução requer uma ação conjunta de produtores, agências governamentais, processadores de alimentos e cientistas (SYDENHAM et al., 1996; BRYDEN, 2007).No Brasil, a RDC oo 7 de 18 de fevereiro de 2011 dispõe sobre limites máximos tolerados (LMT) para micotoxinas em alimentos, para aflatoxinas B1, B2, G1, G2 em amostras de castanhas exceto castanha-do-Brasil, incluindo nozes, pistaches, avelãs e amêndoas é de $10 \mu \mathrm{g} \mathrm{kg}^{-1}$ (BRASIL, 2011).

O monitoramento de fungos pode ser realizado pela quantificação de bolores e leveduras por meio do método de contagem padrão em placas, determinando-se o número de unidades formadoras de colônias (UFC). O método mais recomendado é o plaqueamento em superfície, para aumentar a exposição ao oxigênio e evitar o "stress" causado pelo meio de cultura quente (Silva et al., 2010). A identificação do gênero dos fungos é feita pela confecção de lâminas e observação das estruturas fúngicas em microscópio óptico comparando com as características morfológicas descritas em literatura específica (BARNETT \& HUNTER, 1972). 
Em relação às micotoxinas, as mesmas podem ser quantificadas por cromatografia líquida, gasosa e camada delgada. Outra alternativa são os métodos rápidos que apresentam grandes vantagens como boa sensibilidade, rapidez e baixo custo. Estas técnicas, devido à sua simplicidade analítica, não requerem analistas especializados, entretanto, por apresentarem grandes chances de falsos positivos, sua aplicação está mais relacionada a análises de screening, e posteriormente se utiliza cromatografia líquida como técnica de confirmação dos valores encontrados (MALLMANN et al., 1999).

\subsection{PRESENÇA DE RANÇO}

Quando armazenadas, as macadâmias são suscetíveis ao ranço, que é o resultado da oxidação dos ácidos graxos, o que pode afetar o sabor e odor e consequentemente sua qualidade (COLZATO et al., 2011). Nesse aspecto, a determinação da acidez poderá fornecer um dado importante na avaliação da conservação da macadâmia, uma vez que a rancidez quase sempre é acompanhada pela formação de ácidos graxos livres. Estes são geralmente expressos em índice de acidez ou em \% de ácido oleico (BRASIL, 2005).

Outra maneira de quantificar a rancidez é por meio da avaliação do índice de peróxido, uma vez que os peróxidos são resultantes da oxidação lipídica (BRASIL, 2005). Os principais produtos finais da oxidação lipídica compreendem os derivados da decomposição de hidroperóxidos, como álcoois, aldeídos, cetonas, ésteres e outros hidrocarbonetos (FERRARI, 1998). Ribeiro et al. (1993) realizou um estudo que avaliava o comportamento lipídico de castanhas do Pará armazenadas com e sem casca, em diferentes temperaturas, por 4 meses. Os autores observaram que as castanhas, com casca, mantidas a temperatura ambiente obtiveram formação de peróxido a partir do segundo mês e as mantidas em temperaturas $2^{\circ} \mathrm{C}$ e $-15^{\circ} \mathrm{C}$ tiveram reação mais lenta. Ao final do experimento o índice de peróxido se mostrou praticamente o mesmo, exceto por pequenas modificações que não interferiram na qualidade do produto. Já as castanhas sem casca mostraram um maior grau de oxidação lipídica ainda que $\mathrm{o}$ armazenamento em baixas temperaturas tenha amenizado este efeito. 


\subsection{ANÁLISE SENSORIAL}

Paralelamente à quantificação de rancidez o monitoramento da qualidade sensorial pode ser realizado por meio de testes discrimativos ou afetivos. Os testes discriminativos determinam se existe diferença perceptível entre as amostras de um produto. Dentre seus diversos usos são aplicados para determinar alguma alteração dos atributos sensoriais devido ao tempo de armazenamento. Os testes afetivos acessam diretamente a opinião (preferência ou aceitabilidade) do consumidor sobre características específicas do produto. Dentre suas diversas aplicações pode-se citar a avaliação da aceitabilidade do produto em função do tempo de armazenamento (Ferreira et al., 2000).

\section{CONSIDERAÇÕES FINAIS}

A falta de informações sobre a manutenção da qualidade das nozes de macadâmia,quando armazenadas nas propriedades, impedem que os produtores nacionais obtenham maiores lucros e acelerem a expansão dos cultivos. Análises de quantificação e identificação de fungos e micotoxinas, bem como as análises de índice de acidez, índice de peróxido e análises sensoriaispodem ser indicadas para o monitoramento da qualidade das macadâmias durante o seu armazenamento. Dessa maneira, pode-se determinar o período de conservação das nozes e indicar se houve ou não boas condições de armazenamento. Assim, estudos que envolvam o monitoramento da qualidade das nozes de macadâmia por essas análises são recomendados e podemcolaborar na expansão da cadeia da noz-macadâmia no Brasil.

\section{REFERÊNCIAS}




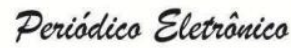 \\ Fórum Ambiental}

da Alta Paulista
Volume 11, Número 02, 2015

Campo, Agronegócio

e as Práticas Sustentáveis

APARECIDO, L.E.; ROLIM, G. de S.; SOUZA, P.S. de. Épocas de florescimento e colheita da nogueira-macadâmia para áreas cafeícolas da região sudeste. Revista Brasileira de Fruticultura, v.36, p.170-178, 2014.

BARNETT, H.L.; HUNTER, B.B. Illustrated genera of imperfect fungi. 3 ed. Burgess Publishing Company. Minneapolis, Minnesota, 1972. 241p.

BOAS, N.V.; CASARIN, J.; CAETANO, J.; GONSALVES JR., A.C.; TARLEY, C.R.T.; DRAGUNSKI, D.C. Biossorção de cobre utilizando-se o mesocarpo e o endocarpo da macadâmia natural e quimicamente tratados. Revista Brasileira de Engenharia Agrícola Ambiental, v.16, n.12, p.1359-1366, 2012.

BRASIL. Ministério da Saúde. Agência Nacional de Vigilância Sanitária. Série A. Normas e Manuais Técnicos: Métodos Físico-químicos para Análise de Alimentos. Brasília, DF, 2005. 1018 p.

BRASIL. Ministério da Saúde. Agência Nacional de Vigilância Sanitária. Resolução no7 de 18 de Fevereiro de 2011. Dispõe sobre os limites máximos tolerados para micotixnas em alimentos. Diário Oficial [da] União, Brasília, 22 fev. 2011. Seção 1, p.72.

BRENES, G.C. El cultivo de la macadamia. San Jose: Editorial Cafesa, 1983. 75p.

BRYDEN, W.L. Mycotoxins in the food chain: human health implications. Asia Pacific Journal of Clinical Nutrition, Austrália, v. 16, suplemento 1, p. 95-101, 2007.

CATI - COORDENADORIA DE ASSISTÊNCIA TÉCNICA INTEGRAL.

Levantamento das unidades de produção agropecuária, estatísticas agrícolas, Estado de São Paulo, 2007/2008, Projeto LUPA. Disponível em:

<http://www.cati.sp.gov.br/projetolupa>. Acesso em: 10 abr. 2012.

CHITARRA, M.I.F.; CHITARRA, A.B. Pós-colheita de frutos e hortaliças: fisiologia e manuseio. 2 ed. Lavras: UFLA, 2005. 785p.

COLZATO, M.; SCRAMIN, J.A.; FORATO, L.A.; COLNAGO, L.A.; ASSIS, O.B.G. H NMR Investigation of oil oxidation in macadamia nuts coated with zein-based films. Journal of Food Processing and Preservation, v.35, p.790-796, 2011.

FERRARI, Carlos Kusano Bucalen. Oxidação lipídica em alimentos e sistemas biológicos: mecanismos gerais e implicações nutricionais e patológicas. Rev. Nutr., Campinas , v. 11, n. 1, p. 3-14, jun. 1998 . Disponível em $<$ http://www.scielo.br/scielo.php?script=sci_arttext\&pid=S1415$52731998000100001 \&$ Ing=pt\&nrm=iso>. Acesso em: 02 jun. 2015.

FERREIRA, V.L.P.; ALMEIDA, T.C.A; PETTINELLI, M.L.C.V.; SILVA, M.A.A.P.; CHAVES, J.B.P.; BARBOSA, E.M.M. Análise sensorial: testes discriminativos e afetivos. Campinas: SBCTA, 2000.127p. 


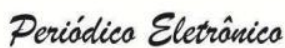 \\ Fórum Ambiental}

da Alta Paulista
Volume 11, Número 02, 2015

Campo, Agronegócio

e as Práticas Sustentáveis

GARBELINI, R.C.B.S. Reguladores vegetais na emergência e desenvolvimento de plantas de macadamia (Macadamia integrifolia Maiden e Betche). 2009. 94p. Tese (Doutorado em Ciências Biológicas) - Faculdade de Ciências Agronômicas, Universidade Estadual Paulista, Botucatu, 2009.

HUETT, D.O. Macadamia physiology review: a canopy light response study and literature review. Australian Journal of Agricultural Research,v.55, n.6, p.609624, 2004.

MCFADYEN, L.M.; ROBERTSON, D.; SEDGLEY, M.; KRISTIANSEN, P.; OLESEN, T. Post-pruning shoot growth increases fruit abscission and reduces stem carbohydrates and yield in macadamia. Annals of Botany, v.107, n.6, p.993-1001, 2011.

MALLMANN, Carlos A.; SANTURIO, Janio M.; DILKIN, Paulo. Equine leukoencephalomalacia associated with ingestion of corn contaminated with fumonisin B1. Rev. Microbiol., São Paulo, v. 30, n. 3, p. 249-252, jul. 1999. Disponível em: <http://www.scielo.br/scielo.php?script=sci_arttext\&pid=S000137141999000300011 \&lng=pt\&nrm=iso >. Acesso em 25 jun. 2015.

MARO, L.A.C.; PIO, R.; PENONI, E.S.; OLIVEIRA, M.C.; PRATES, F.C.; LIMA, L.C.O.; CARDOSO, M.G. Caracterização química e perfil de ácidos graxos em cultivares de nogueira-macadâmia. Ciência Rural, v. 42, n.12, p.2166-2171, 2012.

MARTIN, N.B. Análise do potencial de competição da produção da noz macadâmia em São Paulo e no Havaí. Informações Econômicas, v.22, n.1, p.9-53, 1992.

PERDONÁ, M.J.; SORATTO, R.P. Irrigation and Intercropping with Macadamia Increase Initial Arabica Coffee Yield and Profitability. Agronomy Journal, v.107, n.2, p. 1-12, 2015a.

PERDONÁ, M.J.; SORATTO, R.P. Higher yield and economic benefits are achieved in the macadamia crop by irrigation and intercropping with coffee. Scientia Horticulturae (2015), v. 185, p. 59-67.

PERDONÁ, M.J.; SORATTO, R.P.; ESPERANCINI, M. S. T. Desempenho produtivo e econômico do consórcio de cafeeiro arábica e nogueira-macadâmia. Pesquisa Agropecuária Brasileira, v.50, n.1, p.12-23, 2015.

PIMENTEL, L.D. A cultura da macadâmia. Revista Brasileira de Fruticultura, v.29, n.3, p.414-416, 2007.

PIMENTEL, L.D.; SANTOS, C.E.M. dos; WAGNER JÚNIOR, A.; SILVA, V.A.; BRUCKNER, C.H. Estudo de viabilidade econômica na cultura da noz-macadâmia no Brasil. Revista Brasileira de Fruticultura, v.29, p.500-507, 2007. Disponível em: <http://www.scielo.br/scielo.php?script=sci_arttext\&pid=S010029452007000300018\&lng=en\&nrm=iso>. Acesso em 01 Jun. 2015. 
PIO, Rafael et al. Produção e amplitude de colheita de cultivares de nogueiramacadâmia em Itapira, São Paulo. Rev. Ceres, Viçosa, v. 59, n. 6, p. 826831, Dec. 2012. . Disponível em: <http://www.scielo.br/scielo.php?script=sci_arttext\&pid=S0034737X2012000600013\&lng=en\&nrm=iso >. Acesso em 01 Jun. 2015.

RIBEIRO, M.A.A. et al. Armazenamento da castanha do pará com e sem casca: efeito da temperatura na resistência ao ranço. Sci. agric. (Piracicaba, Braz.), Piracicaba, v. 50, n. 3, p. 343-348, dez. 1993 . Disponível em: $<$ http://www.scielo.br/scielo.php?script=sci_arttext\&pid=S010390161993000300004\&lng=pt\&nrm=iso >. Acesso em: 02 jun. 2015.

SILVA N.; JUNQUEIRA V. C. A.; SILVEIRA N. F. A.; TANIWAKI M. H. ; SANTOS R. F. S. ; GOMES, R. A. R. Manual de métodos de análise microbiológica de alimentos e água. 4 ed. São Paulo: Livraria Varela, 2010. 362p.

SACRAMENTO, C.K.; PEREIRA, F.M.; PERECIN, D.; SABINO, J.C. Capacidade combinatória para frutificação em cultivares de nogueira-macadâmia. Pesquisa Agropecuária Brasileira, v.34, n.11, p. 2045-2049, 1999.

SCHNEIDER, L.M.; ROLIM, G. S.; SOBIERAJSKI, G. R.; PRELA-PANTANO, A.; PERDONÁ, M.J. Zoneamento agroclimático de nogueira-macadamia para o Brasil. Revista Brasileira de Fruticultura, v.34, n.2, p.515-524, 2012.

SILVA, F.A.; MAXIMO, G.J.; MARSAIOLI JR, A.; SILVA, M.A.A.P. Impacto da secagem com microondas sobre o perfil sensorial de amêndoas de noz macadâmia. Ciência e Tecnologia de Alimentos, v.27, n.3, p.553-561, 2007.

SOBIERAJSKI, G.R.; BARBOSA, W.; BETTIOL NETO, J.E.; CHAGAS, E.A.; CAMPO-DALL'ORTO, F.A. Caracterização dos estágios fenológicos em sete cultivares e seleções de nogueira-macadâmia. Revista Brasileira de Fruticultura, v.29, n.3, p.690-694, 2007.

SPONCHIATO, D. Uma castanha por dia. Revista Saúde, v.298, p.18-23, 2008.

SYDENHAM, E. W.; SHEPARD, G. S.; THIEL, P.G.; STOCKENSTROM, S.; SNIJAMAY, P.W.; VAN SCHALKWYK, D. J.(1996) Liquid chromatographic determination of fumonisins B1, B2, and B3 in corn: AOAC-IUPAC collaborative study . Journal of AOAC International, v. 79, n.3, p. 688-696.

WALTON, D.A.; WALLACE, H.M. Quality changes in macadamia kernel between harvest and farm-gate. Journal Science Food Agriculture, v.91, p.480-484, 2011. 